\title{
Empirical Performance Models for P2P and Two Hops Multi-hop Cellular Networks with Mobile Relays
}

\author{
Baldomero Coll Perales ${ }^{+}$, Javier Gozalvez ${ }^{+}$and Joaquin Sanchez-Soriano ${ }^{\dagger}$ \\ 'UWICORE Laboratory, 'Operations Research Center \\ Miguel Hernandez University of Elche \\ Avda. de la Universidad, s/n, 03202, Elche (Spain) \\ +34 965222031 \\ bcoll@umh.es, j.gozalvez@umh.es, joaquin@umh.es
}

\begin{abstract}
Multi-hop Cellular Networks using Mobile Relays (MCN-MR) are being studied to overcome certain limitations of traditional single hop cellular systems through the integration of cellular and Peer to Peer (P2P) ad-hoc communications. Current MCN-MR research is generally analytical or simulation based, and there is yet the need for studies that empirically demonstrate the potential of MCN-MR and provide valuable models for the research community. In this context, this paper presents the first 2-hop MCN-MR empirical performance models derived from field tests over live networks. The MCN-MR performance is measured for downlink transmissions as the throughput experienced by mobile users. The model parameters are shown to depend on the distance, Line-Of-Sight (LOS) or Non-LOS (NLOS) conditions, and communication settings of the ad-hoc link. As a result, the paper also reports a set of distance-based empirical P2P link level models.
\end{abstract}

\section{Categories and Subject Descriptors}

C.1.3 [Processor Architectures]: Other Architecture Styles cellular architecture (e.g., mobile), heterogeneous (hybrid) systems. C.2.1 [Computer-Communication Networks]: Wireless communication. C.4 [Performance of Systems]: Measurement techniques, Modeling techniques.

\section{General Terms}

Measurement, Performance, Experimentation.

\section{Keywords}

Multi-hop cellular networks; model; mobile relay; peer to peer (P2P).

\footnotetext{
Permission to make digital or hard copies of all or part of this work for personal or classroom use is granted without fee provided that copies are not made or distributed for profit or commercial advantage and that copies bear this notice and the full citation on the first page. Copyrights for components of this work owned by others than ACM must be honored. Abstracting with credit is permitted. To copy otherwise, or republish, to post on servers or to redistribute to lists, requires prior specific permission and/or a fee. Request permissions from from permissions@acm.org.

$P M^{2} H W^{2} N^{\prime} 13$, November 3-8, 2013, Barcelona, Spain.

Copyright 2013 ACM 978-1-4503-2371-0/13/11 ..\$15.00.

http://dx.doi.org/10.1145/2512840.2512844
}

\section{INTRODUCTION}

Single-hop cellular systems have difficulty in providing homogeneous Quality of Service (QoS) levels across the cell area due to the effect of distance and surrounding obstacles on the direct link established between Mobile Stations (MSs) and Base Stations (BSs). An alternative to overcome this limitation is the integration of cellular and P2P ad-hoc technologies into what is usually referred as Multi-hop Cellular Networks (MCNs). MCN networks substitute the direct MS-BS link by multiple hops using either Fixed Relays (MCN-FRs) or Mobile Relays (MCN-MRs). The introduction of relaying techniques into cellular standards has initially focused in MCN-FR solutions [1], although MCN-MR networks are gaining significant research attention [2-3]. In this context, it is interesting noting the $3 \mathrm{GPP}$ activities towards the definition of proximity-based communications that will allow users in proximity to directly communicate with each other (not through the BS) [4]. The study activities also cover 2-hop MCNMR communications between a BS and an end user using $\mathrm{P} 2 \mathrm{P} / 802.11$-enabled mobile users as relays ('ProSe UE-toNetwork Relay' in [4]). MCN-MR and proximity-based communications can offer numerous advantages in terms of capacity enhancement, radio cell extension, lower infrastructure deployment cost, power saving and energy efficiency (in particular for delay tolerant services), network offloading, and flexible support for public safety services among others [3-4].

The design of future MCN-MR systems will require investigating advanced communication and networking protocols that efficiently exploit the contact opportunities between mobile devices. Important aspects to investigate include, among others, the selection of forwarding nodes, the design of opportunistic networking schemes for delay tolerant services, or the implementation of mode selection techniques capable to identify when and for which mobiles a MCN-MR connection is more beneficial than a traditional single-hop cellular one. This type of investigations is generally conducted through analytical or simulation-based studies that could be enhanced and facilitated if MCN-MR performance models capturing the performance dependencies present in real systems would be available. For example, the models could help selecting forwarding nodes that guarantee the target end-to-end performance. MCN-MR performance models could also facilitate the design of efficient mode selection mechanisms capable to identify the conditions under which MCN-MR would be a viable communications alternative in heterogeneous wireless networks. In this context, this paper goes a step beyond the current state of the art, and presents, to the authors' knowledge, the first MCN-MR empirical performance models obtained from an urban field measurement campaign using live networks. The presented models focus on 2- 
hop MCN-MR communications, and differentiate between LOS and NLOS conditions between the forwarding and destination nodes. The MCN-MR performance modeling reported in this paper shows that the MCN-MR performance strongly depends on the $\mathrm{P} 2 \mathrm{P}$ ad-hoc conditions and quality. As a result, the paper also presents empirical P2P link level models (under LOS and NLOS conditions) based on the distance between nodes.

\section{RELATED WORK}

Previous studies have reported the advantages that MCN-MR networks could provide over traditional cellular architectures. However, these studies are generally analytical or simulation based, and therefore cannot completely capture the performance dependencies present in real systems. The first experimental evaluation of MCN technologies is reported in [5] where the authors present a Long Term Evolution (LTE)-Advanced prototype using fixed relays. The conducted experiments demonstrate that MCN-FR systems can significantly improve the coverage and capacity of cellular systems. Similar results are shown in [6] using an LTE Time Division Duplex (TDD) in-band FR prototype. The conducted measurements allowed identifying the need to strategically locate FR nodes at the cell edge under LOS conditions with the BS in order to maximize capacity and end-user QoS. The work presented in [5] also showed the impact of the distance and operating conditions between the fixed relay and the mobile user on the end-to-end performance.

Recent studies have looked at the potential of mobile devices. For example, [7] introduced the Cool-Tether platform designed to study the energy performance of a proposed mixed cellular and 802.11 architecture. The study demonstrates that the reverse infrastructure-mode adopted in Cool-Tether minimizes energy consumption of mobile devices, but does not analyze the end-toend quality of service performance. Another interesting development is Qualcomm's FlashLinQ [8] technology. The technology, now being evolved into LTE-Direct, enables mobile devices to automatically discover neighboring terminals and communicate with them (with/without infrastructure support). This type of device-to-device technologies, once designed and implemented, will represent an important step towards future MCN-MR systems. An experimental demonstration of the potential of MCN-MR technologies has only been recently presented by the authors in [9]. In [9], the authors quantify and validate the benefits that MCN-MR can provide over traditional cellular systems in terms of extended radio coverage, reduced energy consumption, and improved QoS at large distances to the serving BS, in indoor environments, under NLOS propagation conditions, and while crossing handover areas. Thanks to the availability of a unique MCN-MR experimental testbed, the authors go a step beyond in this study, and present the first MCNMR empirical performance models obtained from an urban field measurement campaign using live networks. The employed testbed allows geo-referencing the cellular and P2P ad-hoc performance measurements using GPS devices (Bluetooth and USB) connected to the mobile nodes. The GPS logged data has been essential to derive distance-based models of the end-user MCN-MR performance and the P2P ad-hoc link level quality.

\section{MCN-MR FIELD TESTS}

\subsection{Testing Environment}

A large set of field trials has been conducted in the city of Elche (Spain) using Orange's live cellular network and the mHOP testbed [9]. Figure 1 illustrates the 2-hop MCN-MR testing environment, which includes a static Hybrid Node (HN) and a mobile Destination Node (DN) experiencing LOS or NLOS conditions with the HN. The communications link with the BS is established through Orange's HSDPA network, while the ad-hoc link uses IEEE $802.11 \mathrm{~g}$ technology at $2.4 \mathrm{GHz}^{1}$.

The HN acts as a gateway between the cellular and ad-hoc links. The HN is implemented in a Linux standard laptop using a Nokia 6720c terminal with Nemo Handy (http://www.anite.com) as modem for the HSDPA (release 5) cellular link. In addition to their built-in wireless interface (used for monitoring transmitted and received packets), the laptops have been equipped with a Wireless ExpressCard for the ad-hoc transmissions (a virtual interface of this external card is also used for monitoring). The Wireless ExpressCard is controlled by the Ath9k driver that implements adaptive schemes to dynamically modify the $802.11 \mathrm{~g}$ ad-hoc data rate and transmission power based on the link quality conditions (additional field tests were conducted fixing the data rate and the transmission power). The $\mathrm{HN}$ implements modified routing tables in order to forward in real-time the data received from the cellular link to the 802.11 ad-hoc link. The HN also incorporates two GPS receivers for the cellular and 802.11 interfaces. The Linux libraries libpcap and libgps have been used to develop a sniffing and a GPS application respectively. The sniffing application continuously monitors the 802.11 performance, while the GPS application allows tracing the position of the node and geo-referencing all the logged radio measurements with a periodicity of $1 \mathrm{~Hz}$. The mobile DN includes the same functionalities as the $\mathrm{HN}$ except for the cellular modem.

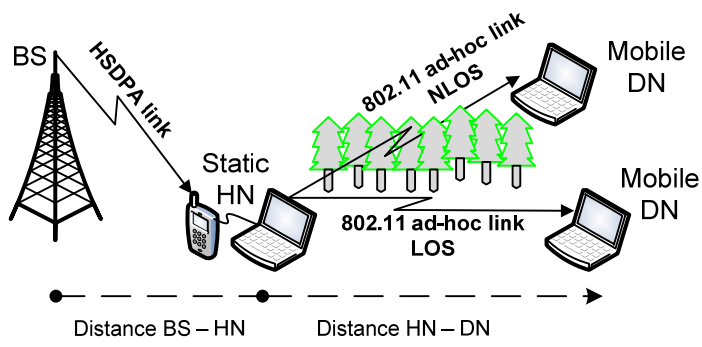

Figure 1. Field testing environment.

The field tests were conducted in a four-lane avenue close to the University. This avenue ensured LOS conditions between the HN and the mobile DN when both were located at the same side of the avenue. The NLOS conditions were achieved locating the HN and the mobile DN in opposite sidewalks of the avenue, with vehicles continuously crossing over and dense vegetation in the avenue's median. The HN remains in a static position during the tests (tests for different locations of the $\mathrm{HN}$ have been conducted). The

\footnotetext{
${ }^{1}$ An objective of this study was to derive the models using live cellular networks. At the time of conducting this study, no LTE deployment was active in Spain. Ad-hoc P2P connections were established using 802.11 technologies since cellular device-todevice solutions are not yet available, or even standardized.
} 
mobile DN moves away from the location of the HN until the 802.11 ad-hoc link with the HN is lost. During the tests, UDP traffic is downloaded from a server managed and located at the UWICORE lab. UDP traffic was selected since it allows identifying the radio link that represents the bottleneck of a multihop transmission. In addition, UDP traffic maximizes the transmission data rate since the datagrams sent by the traffic source do not require an end-to-end acknowledgment [10].

\subsection{MCN-MR Performance}

The MCN-MR performance is measured in terms of the throughput experienced by the mobile DN. Initial tests showed that the HSDPA radio resources assigned to mobile users depend on their channel quality. However, the percentage of time that these resources were actually been used by each user (usage) depends on the operator's radio resource management policy. For example, the conducted measurements showed that under certain conditions, the network assigned more codes to a user at the cell edge experiencing bad link quality than to a user close to the BS. This policy resulted in that both users obtained similar throughput levels despite their different link quality conditions. To reduce the impact of resource management policies, and capture the cellular performance dependence with the channel quality, the MCN-MR throughput is here computed as the ratio between the measured MCN-MR user throughput and the usage of the cellular resources [9].

Figure 2 shows, for one of the conducted tests, the MCN-MR throughput experienced by the mobile DN when its 802.11 ad-hoc link with the HN operates under LOS (Figure 2.a) and NLOS (Figure 2.b) conditions. The MCN-MR throughput is depicted as

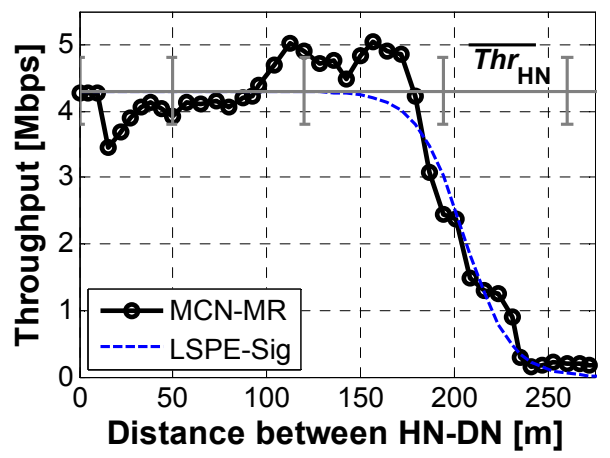

a) LOS conditions between HN-DN



b) NLOS conditions between HN-DN

Figure 2. MCN-MR throughput experienced by the DN as a function of the distance between the static $\mathrm{HN}$ and the mobile DN. The HN is located 500 meters away from the BS. a function of the distance between the $\mathrm{HN}$ and the DN (the DN begins the test close to the $\mathrm{HN}$ ). The reported measurements are averaged over time period of 1 second given the GPS updating rate $(1 \mathrm{~Hz})$. Figure 2 also represents the average throughput of the HSDPA cellular link measured at the $\mathrm{HN}\left(\overline{T h r_{\mathrm{HN}}}\right)$ and its standard deviation. The depicted results show that the MCN-MR throughput at the mobile DN is first upper-bounded by the cellular performance at the HN. However, the MCN-MR throughput decreases with the distance, with the reduction starting at smaller distances to the HN under NLOS conditions than under LOS. This is due to the more challenging NLOS propagation conditions, and the consequent lower received signal levels. The distance at which the MCN-MR throughput operates below $\overline{T h r_{\mathrm{HN}}}$ is referred in the paper as the critical distance. A large set of field tests have been conducted for different locations of the HN (200, 500 and $800 \mathrm{~m}$ ). Varying the location of the HN resulted in different $\overline{T h r_{\mathrm{HN}}}$ and MCN-MR throughput at the DN. However, similar trends to that depicted in Figure 2 were always appreciated.

\section{MCN-MR PERFORMANCE MODELING}

As previously mentioned, all field tests exhibited similar performance trends as those shown in Figure 2. The performance curve has a symmetric $\mathrm{S}$ shape. There are several mathematical functions that model such shape, and can be grouped into three broad categories: exponential, piecewise-defined, and sigmoid functions. To model the MCN-MR throughput performance at the $\mathrm{DN}$, a function from each of these categories has been selected. More complex functions can also provide good fits, but the selected ones provide a reasonable trade-off between accuracy and analytical-simulation tractability. In particular, the following functions have been selected:

-Exp function:

$$
\operatorname{Exp}\left(p_{1}, p_{2}, d\right)=A \cdot e^{-\left(p_{1} \cdot d\right)^{p_{2}}}, \forall d \in\{0, \ldots, M\}
$$

-Part function:

$$
\operatorname{Part}\left(p_{1}, p_{2}, d\right)= \begin{cases}A & d<p_{1} \\ k \cdot\left(\frac{1}{d}-\frac{1}{p_{2}}\right) & p_{1} \leq d<p_{2}, \forall d \in\{0, \ldots, M\} \\ 0 & p_{2} \leq d\end{cases}
$$

where $k=A /\left(1 / P_{1}-1 / P_{2}\right)$

-Sig function:

$$
\operatorname{Sig}\left(p_{1}, p_{2}, d\right)=A \cdot\left[1-\frac{1}{1+e^{-p_{1} \cdot\left(d-p_{2}\right)}}\right], \forall d \in\{0, \ldots, M\}
$$

$d$ represents the distance in meters between the static $\mathrm{HN}$ and the mobile DN, and $A$ the upper asymptote or $\overline{T h r_{\mathrm{HN}}} \cdot p_{1}$ and $p_{2}$ are the variables or fitting parameters that modify the characteristics of the S-shaped functions: (i) length of the upper asymptote (i.e. distance at which the MCN-MR throughput starts decreasing or critical distance), (ii) slope of the curve (i.e. how fast the MCNMR throughput degrades), and (iii) the start of the lower asymptote (i.e. distance at which the MCN-MR connectivity is lost). The $\left(p_{1}, p_{2}\right)$ parameters of the Part function refer to the (i) and (iii) characteristics. The relation between $\left(p_{1}, p_{2}\right)$ and the characteristics of the S-shaped function is not so direct for the Exp and Sig functions [11]. 
The curve fitting technique has been selected to derive the fitting parameters that minimize the residue/error between the measured data and the proposed modeling functions. The curve fitting techniques distinguish themselves by the estimator used to calculate the error. The least-square estimator produces the maximum-likelihood estimate of the parameters when the errors are distributed all along the modeling function, as it is the case of the MCN-MR field measurements here reported. As a result, the Least-Square Parameter Estimation (LSPE) technique has been used to derive the fitting parameters $\left(p_{l}, p_{2}\right)_{j}$ for each of the selected functions $(j \in\{\mathrm{Exp}$, Part, Sig $\})$. The error $(e)$ is computed following the mean of the least-square estimator:

$$
e_{j}=\sqrt{\frac{\sum_{d=0}^{M}\left(f_{j}\left(p_{1}, p_{2}, d\right)-g(d)\right)^{2}}{M}}
$$

where $f_{j}\left(p_{1}, p_{2}, d\right)$ represents the modeling functions and $g(d)$ represents the measured data (in this case, the MCN-MR throughput experienced at the mobile DN).

Figure 2 shows that LSPE achieves a good match between the MCN-MR throughput at the mobile DN and the Sig function under LOS and NLOS conditions (a similar match is also obtained between the MCN-MR throughput at the mobile DN and the Part and Exp functions). The same LSPE process has then been conducted for all field tests, which results in a set of fitting parameters $\left(p_{1}^{i}, p_{2}^{i}\right)_{j}$ and errors $e_{j}^{i}(\forall i \in\{1, \ldots \mathrm{N}\}$, with $\mathrm{N}$ representing the number of conducted field tests ${ }^{2}$ ). For each of the selected functions, the obtained results are grouped based on the visibility conditions between the static $\mathrm{HN}$ and the mobile DN, and the different locations of the static HN. Clear differences were appreciated between the $\left(p_{1}, p_{2}\right)$ parameters obtained under LOS and NLOS conditions. However, only small differences were obtained for different locations of the static $\mathrm{HN}^{3}$ despite the fact that the cellular throughput at the $\mathrm{HN}$ varies with the distance to the BS (Figure 3). As a result, the $\left(p_{1}, p_{2}\right)$ parameters for the different locations of the static $\mathrm{HN}$ have been grouped.

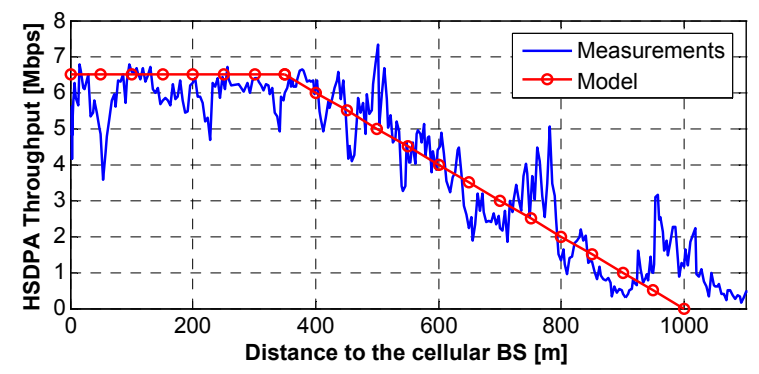

Figure 3. HSDPA throughput measurements with the distance.

Table 1. 2-hop MCN-MR $\left(p_{1}, p_{2}\right)$ parameters

\begin{tabular}{|c|c|c|c|c|}
\hline \multirow{2}{*}{ Function } & \multicolumn{2}{|c|}{ LOS } & \multicolumn{2}{c|}{ NLOS } \\
\cline { 2 - 5 } & $\left(\boldsymbol{p}_{\mathbf{1}}, \boldsymbol{p}_{\mathbf{2}}\right)$ & $\begin{array}{c}\text { Error } \\
{[\mathbf{M b p s}]}\end{array}$ & $\left(\boldsymbol{p}_{\boldsymbol{1}}, \boldsymbol{p}_{\mathbf{2}}\right)$ & $\begin{array}{c}\text { Error } \\
{[\mathbf{M b p s}]}\end{array}$ \\
\hline Exp & $(0.005,12.05)$ & 0.644 & $(0.012,6.53)$ & 0.440 \\
\hline Part & $(157.9,230.8)$ & 0.643 & $(60.6,113.8)$ & 0.448 \\
\hline Sig & $(0.12,186.99)$ & 0.633 & $(0.12,79.67)$ & 0.436 \\
\hline
\end{tabular}

\footnotetext{
${ }^{2}$ In total, more than 60 tests were conducted.

${ }^{3}$ For example, the difference was smaller than $10 \mathrm{~m}$ for the Part function.
}

Table 1 summarizes the average of the resulting fitting parameters $\left(p_{1}, p_{2}\right)$ for the selected functions under LOS and NLOS conditions of the ad-hoc link. Table 1 also depicts the average of the errors between the selected functions and the measured data under LOS and NLOS conditions when using the resulting fitting parameters. The results reported in Table 1 show that the Sig function provides the lower error under LOS and NLOS conditions. As a result, this study proposes to model the 2-hop MCN-MR throughput performance experienced at a mobile DN using the Sig function reported in (5) with the fitting parameters shown in Table 1 under LOS and NLOS conditions. For the Sig function's fitting parameters, the critical distance is equal to 156 meters and 52 meters under LOS and NLOS conditions respectively. In (5), $d$ represents the $\mathrm{HN}-\mathrm{DN}$ separation distance.

$$
\operatorname{MCN}-M R T h r\left(p_{1}, p_{2}, d\right)_{2 \text {-hop DN }}=\overline{T h r_{\mathrm{HN}}} \cdot\left[1-\frac{1}{1+e^{-p_{1} \cdot\left(d-p_{2}\right)}}\right]
$$

The $\overline{T h r_{\mathrm{HN}}}$ parameter in (5) represents the average HSDPA throughput measured at the static HN. As shown in Figure 3, its value depends on the actual position of the static $\mathrm{HN}$ and can be modeled as follows ${ }^{4}$ :

$$
\overline{T h r_{\mathrm{HN}}}(d)=\left\{\begin{array}{lll}
6.5 & \text { if } & d<350 \mathrm{~m} \\
-0.01 \cdot d+10 & \text { if } & d \geq 350 \mathrm{~m}
\end{array}\right.
$$

with $d$ representing the distance between the $\mathrm{HN}$ and the serving BS. Those interested in using the 2-hop MCN-MR throughput performance model proposed in (5) could use the $\overline{T h r_{\mathrm{HN}}}$ measurements depicted in Figure 3 and modeled in (6), or use their own cellular measurements. It is important emphasizing that the derived model can be used for different locations of the static $\mathrm{HN}$ since as previously discussed the $\left(p_{1}, p_{2}\right)$ parameters do not depend on $\overline{T h r_{\mathrm{HN}}}$.

\subsection{Validation tests}

To validate the proposed MCN-MR performance model, additional tests were carried out at another location with a different serving BS. Figure 4 shows the MCN-MR performance measured at one of these additional tests, and compares it with the proposed model. The figure shows an adequate matching between the proposed model and the measured performance. For the performed additional tests, the obtained average error was in line with the results reported in Table 1 . In particular, the average error was equal to $0.66 \mathrm{Mbps}$ under LOS conditions and $0.51 \mathrm{Mbps}$ under NLOS conditions. The obtained results show then that the proposed empirical model is capable to adequately model the MCN-MR performance at locations with different morphology and operating conditions.

\subsection{IEEE 802.11g Fixed Transmission Mode}

The previous models were obtained using the default configuration of the $802.11 \mathrm{~g}$ cards used in the mHOP testbed. Such configuration adapts the transmission parameters (data rate and transmission power) based on the experienced link quality conditions. In this context, it is of interest checking whether the 2hop MCN-MR throughput performance models follow similar

4 The HSDPA throughput measurements here reported are computed as the ratio between the measured HSDPA user throughput and the usage of cellular resources. 


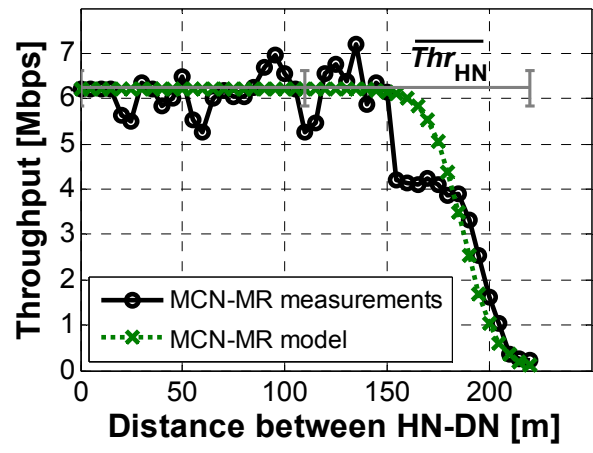

a) LOS conditions between HN-DN

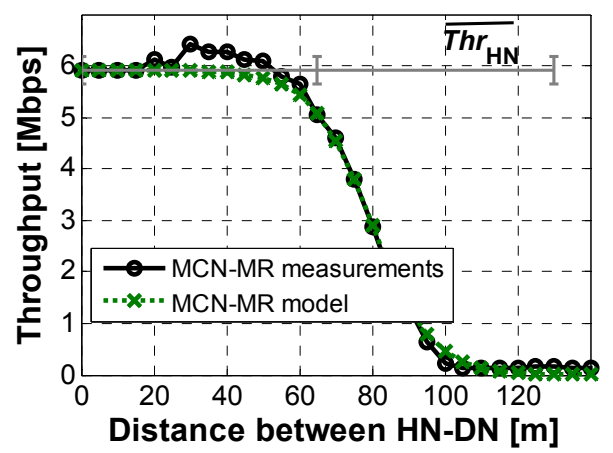

b) NLOS conditions between HN-DN

Figure 4. Validation of the MCN-MR performance model at a different location and serving BS. The HN was located 400 meters away from the BS.

trends when operating with fixed transmission power levels and data rates. Additional field tests have therefore been conducted under similar conditions as those reported in previous sections and illustrated in Figure 1. The IEEE 802.11g transmission power was fixed to $19 \mathrm{dBm}(19 \mathrm{dBm}$ was previously the power limit, but the actual transmission power was dynamically modified) and the data rate to 48 and $54 \mathrm{Mbps}$. The obtained measurements showed indeed similar trends as those observed when the $802.11 \mathrm{~g}$ adaptive scheme was active and represented in Figure 2. As a result, the same LPSE curve fitting process using the Sig function has been applied to these measurements. Table 2 reports the obtained fitting parameters. In this case, the critical distance is equal to 125 (LOS) and 50 (NLOS) meters when the mobile nodes communicate using the $54 \mathrm{Mbps}$ data rate. A $48 \mathrm{Mbps}$ data rate results in a critical distance of 143 (LOS) and 65 (NLOS) meters. In addition, the decreasing slope of the MCN-MR throughput at the DN (related with the $p_{1}$ parameter) is greater when the mobile nodes uses the $54 \mathrm{Mbps}$ data rate than when using the $48 \mathrm{Mbps}$ one. This is due to the more robust modulation and coding scheme used by the $48 \mathrm{Mbps}$ data rate.

Table 2. 2-hop MCN-MR $\left(p_{1}, p_{2}\right)$ parameters with fixed IEEE 802.11g transmission mode

\begin{tabular}{|c|c|c|c|c|}
\hline & \multicolumn{2}{|c|}{$\mathbf{5 4 M b p s - 1 9 d B m}$} & \multicolumn{2}{c|}{$\mathbf{4 8 M b p s - 1 9 d B m}$} \\
\hline & $\left(\boldsymbol{p}_{1}, \boldsymbol{p}_{2}\right)$ & $\begin{array}{c}\text { Error } \\
{[\mathrm{Mbps}]}\end{array}$ & $\left(\boldsymbol{p}_{1}, \boldsymbol{p}_{2}\right)$ & $\begin{array}{c}\text { Error } \\
{[\mathrm{Mbps}]}\end{array}$ \\
\hline LOS & $(0.12,156.01)$ & 0.637 & $(0.07,195.45)$ & 0.672 \\
\hline NLOS & $(0.15,78.93)$ & 0.446 & $(0.13,98.5)$ & 0.373 \\
\hline
\end{tabular}

\section{P2P LINK LEVEL MODELING IN MCN- MR COMMUNICATIONS}

The MCN-MR performance has been shown to strongly depend on the conditions experienced between mobile relays. It is therefore of interest to analyze and characterize the P2P ad-hoc communications between the static $\mathrm{HN}$ and the mobile DN in the 2-hop MCN-MR scenario illustrated in Figure 1. The conducted field tests focused on downlink MCN-MR transmissions from the $\mathrm{BS}$ to the DN. In this context, it is important remembering that the MCN-MR performance is upper-bounded by the cellular throughput, and so is the P2P 802.11 throughput performance. The P2P communications characterization and modeling focuses then on the IEEE 802.11g Data Rate, Packet Error Ratio (PER) and Receive Signal Strength Indicator (RSSI). Other studies have previously modeled different aspects of 802.11 communications. For example, [12] characterizes the performance of Minstrel (the algorithm implemented on the mHOP 802.11 nodes to select the Data Rate) for different transmission power configurations and fixed separation distances between the $802.11 \mathrm{~g}$ devices. PER and RSSI 802.11 models can be found in the literature, although PER models are mainly derived as a function of the packet-length and/or physical-level metrics (e.g. Signal to Noise Ratio) [13-14]. The P2P 802.11 models here presented complement existing models by modeling P2P 802.11 communications as a function of the distance between mobile relays. Characterizing the P2P communications as a function of the distance between mobile relays can facilitate the use of the models in analytical and simulation-based studies.

The IEEE 802.11g Data Rate represents the transmission mode used in the ad-hoc communication between the static $\mathrm{HN}$ and the mobile DN. IEEE $802.11 \mathrm{~g}$ defines twelve possible combinations of modulation and coding schemes that result in the following set

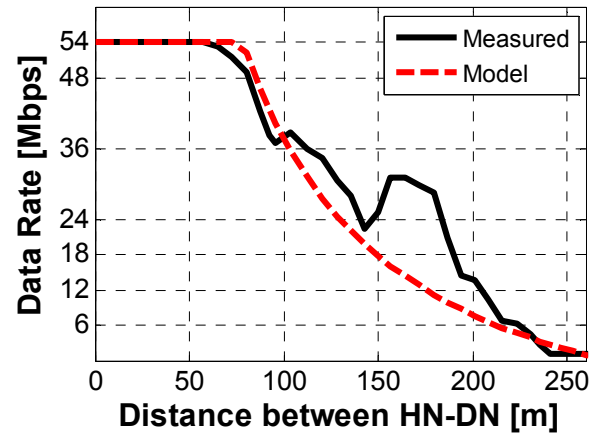

a) LOS conditions between HN-DN

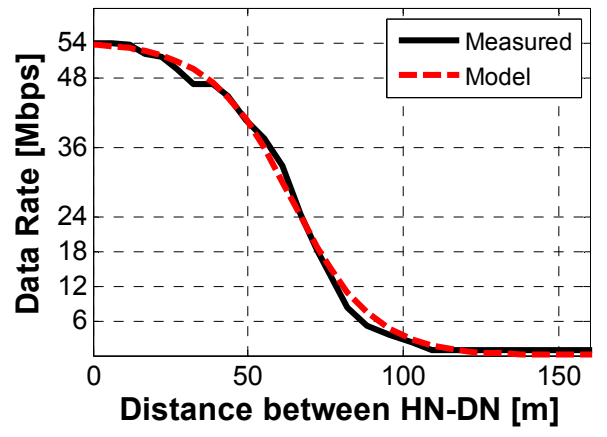

b) NLOS conditions between HN-DN

Figure 5. IEEE 802.11g Data Rate. 
of eligible data rates: $\{54,48,36,24,18,12,9,6 ; 11,5.5,2,1\}$ Mbps. As previously mentioned, the 802.11 card's Ath9k driver dynamically selects the IEEE $802.11 \mathrm{~g}$ Data Rate based on the link quality conditions. Figure 5 shows, for one of the conducted tests, the measured Data Rate when the $802.11 \mathrm{~g}$ ad-hoc link between the HN and mobile DN operates under LOS and NLOS conditions. The Data Rate is depicted as a function of the distance between the static HN and the mobile DN. The depicted results show that the IEEE $802.11 \mathrm{~g}$ Data Rate is initially set to 54Mbps. However, more robust transmission modes are used with the increasing distance between the $\mathrm{HN}$ and the $\mathrm{DN}$. The more challenging NLOS propagation conditions result in the adaptation of the Data Rate at smaller distances, which is consistent with the earlier MCN-MR throughput reduction depicted in Figure 2 under NLOS conditions. The extracted measurements show similar properties to the S-shaped mathematical functions used to model the 2-hop MCN-MR performance. As a result, the LSPE curve fitting process using the Exp, Part and Sig functions has also been applied to derive the $802.11 \mathrm{~g}$ Data Rate models. The smoother pass from the upper asymptote to the slope of the curve (Figure 5.b) results in that the Sig function provides the best match with the IEEE $802.11 \mathrm{~g}$ Data Rate under NLOS conditions. The IEEE 802.11g Data Rate model is therefore represented in (7) with the fitting $\left(p_{1}, p_{2}\right)$ parameters equal to $(0.076,63.97)$ (resulting in an average error equal to $1.3 \mathrm{Mbps}$ ). The measurements reported in Figure 5.a for LOS conditions were better fitted with the Part function. As a result, the IEEE 802.11g Data Rate under LOS conditions is modeled by (8), with the fitting parameters being equal to $(78.47,270.85)$ (resulting in an average error equal to $3.95 \mathrm{Mbps}$ ). In both models (7 and 8$), d$ represents the distance between the $\mathrm{HN}$ and the $\mathrm{DN}$, and the upper asymptote $(A)$ is set to $54 \mathrm{Mbps}$. The derived models are also depicted in Figure 5 showing an adequate match with the measurements.

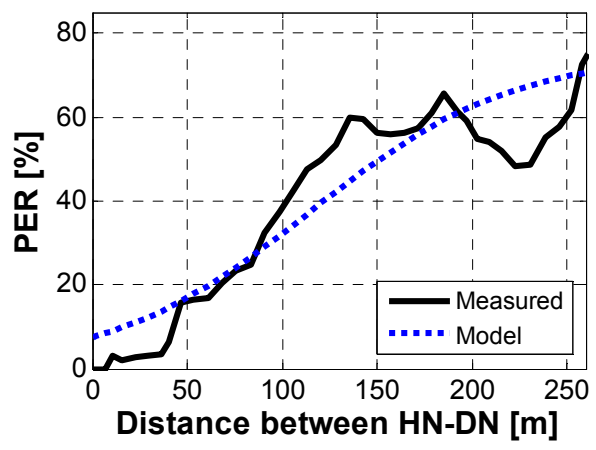

a) LOS conditions between HN-DN



b) NLOS conditions between HN-DN

Figure 6. IEEE 802.11g PER.

$$
\begin{gathered}
\text { DataRate }_{\text {NLOS }}\left(p_{1}, p_{2}, d\right)=54 \cdot\left[1-\frac{1}{1+e^{-p_{1} \cdot\left(d-p_{2}\right)}}\right] \\
\text { DataRate }_{\text {LOS }}\left(p_{1}, p_{2}, d\right)= \begin{cases}54 & k \cdot\left(\frac{1}{d}-\frac{1}{p_{2}}\right) \\
0 & \begin{array}{l}
d<p_{1} \leq d<p_{2} \\
p_{2} \leq d
\end{array}\end{cases}
\end{gathered}
$$

The PER has been measured in the $\mathrm{HN}$ as the ratio between the data packets retransmitted and the total number of data packets transmitted (including retransmissions) [13]. A data packet retransmission is required if the $\mathrm{HN}$ does not receive an $\mathrm{ACK}$ from the DN. This can be caused by an error in the transmission of the data packet from the $\mathrm{HN}$ to the $\mathrm{DN}$ or an error in the ACK sent from the DN to the HN. An example of the PER measured at the $\mathrm{HN}$ as a function of the separation distance between the HN and DN is shown in Figure 6. The obtained measurements show that the PER augments with the distance (both for LOS and NLOS conditions) even if more robust Data Rates are being used with increasing distances (Figure 5). The upper PER limit is reached at the distance at which the MCN-MR throughput measured at the DN is negligible (Figure 2). The PER modeling analysis has considered the original (not symmetric) $\mathrm{S}$ shape of the Sig, Part and Exp functions and the LSPE curve fitting technique. The conducted fitting process resulted in the Sig function providing the lower error between the theoretical functions and the measured data both under LOS and NLOS conditions. The PER is therefore model as shown in (9). The fitting $\left(p_{1}, p_{2}\right)$ parameters are equal to $(0.019,115.15)$ under LOS conditions (resulting in an average error of $9.58 \%$ ) and equal to $(0.0475,54.38)$ under NLOS conditions (resulting in an average error of $5.01 \%$ ). The $d$ parameter in (9) represents the distance between the static HN and the mobile DN. The measured upper PER limit/asymptote $\left(U_{P E R}\right)$ is on average $75 \%$ both under LOS and NLOS conditions. The derived models are also depicted in Figure 6. The worse $802.11 \mathrm{~g}$ ad-hoc performance under NLOS conditions is highlighted by the higher value of $p_{I}$ and the lower value of $p_{2}$. This fact also explains the lower critical distance of the MCN-MR throughput under NLOS conditions.

$$
\operatorname{PER}\left(p_{1}, p_{2}, d\right)=\frac{U_{P E R}}{1+e^{-p_{1} \cdot\left(d-p_{2}\right)}}
$$

The RSSI represents the signal strength at which 802.11g data packets are correctly received at the DN (Figure 7). It is common to represent the average strength of the RSSI in $\mathrm{dBm}$ as a logdistance function (10), where $R S S I_{o}$ is the RSSI at a reference distance $\left(d_{o}\right)$ and $\alpha$ is the RSSI exponent. The fitting $\left(R S S I_{o}, \alpha\right)$ parameters derived using the LSPE curve fitting technique are equal to $(-37.95,1.73)$ for LOS conditions, with an average error of $4.07 \mathrm{dBm}$. The fitting parameters under NLOS were found to be equal to $(-34.88,2.21)$, with an average error of $3.95 \mathrm{dBm}$. For both scenarios, $d_{o}$ is set to $1 \mathrm{~m}$. Using (10), it is possible to detect the RSSI levels characterizing the HN-DN critical distance. For example, in the case of a P2P link (2-hop MCN-MR with adaptive transmission schemes) under LOS conditions, the RSSI level at which the throughput is below $\overline{T h r_{\mathrm{HN}}}$ is found to be equal to -75.8 $\mathrm{dBm}$. Under NLOS conditions, this value is equal to $-72.8 \mathrm{dBm}$.

$$
R S S I\left(R S I_{0}, \alpha, d\right)=R S S I_{0}-10 \alpha \log \left(d / d_{0}\right)
$$




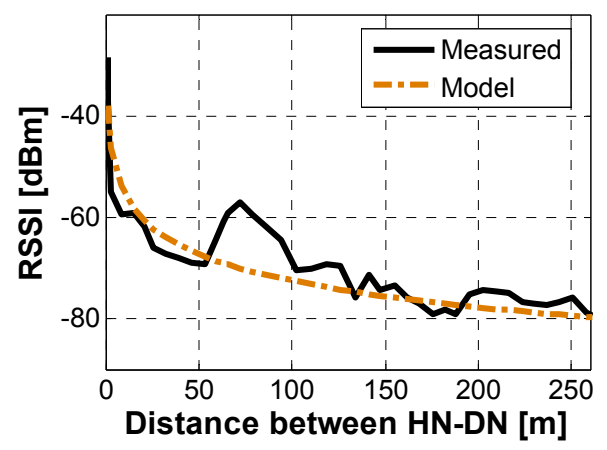

a) LOS conditions between HN-DN

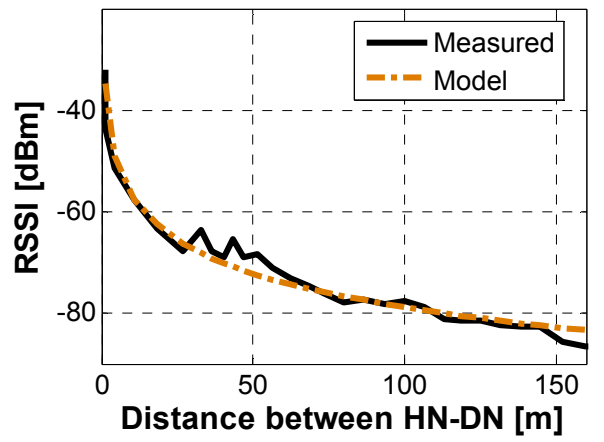

b) NLOS conditions between HN-DN

Figure 7. IEEE 802.11g RSSI.

\subsection{IEEE 802.11g Fixed Transmission Mode}

Using (9) and (10), it is also possible to model the P2P link level communications for a 2-hop MCN-MR connection using $802.11 \mathrm{~g}$ fixed transmission modes. The resulting model parameters are summarized in Table 3. As it can be appreciated, using the data rate and transmission power configuration (54Mbps-19dBm) results in higher PER values than using the (48Mbps-19dBm) configuration; this is reflected in the higher values of $U_{P E R}$ and $p_{l}$. The use of adaptive schemes for the P2P connection results in lower $U_{P E R}$ and $p_{I}$ values. Fixing the transmission power to $19 \mathrm{dBm}$ results in that the RSSI fitting parameters do not show significant differences under similar propagation conditions (despite using a different Data Rate). However, the worse NLOS conditions are captured in the RSSI fitting parameters. The comparison with the RSSI fitting parameters obtained with adaptive schemes reveal the higher signal strength levels measured when fixing the transmission power to $19 \mathrm{dBm}$. Although $19 \mathrm{dBm}$ is the power limit when using adaptive schemes, the transmission power is dynamically modified based on the link quality conditions.

Table 3. PER and RSSI $\left(p_{1}, p_{2}\right)$ parameters with fixed IEEE 802.11g transmission mode and power

\begin{tabular}{|c|c|c|c|c|c|}
\hline & \multicolumn{2}{|c|}{ 54Mbps - 19dBm } & \multicolumn{2}{|c|}{ 48Mbps - 19dBm } \\
\hline & & $\left(p_{1}, p_{2}\right)$ & Error & $\left(p_{1}, p_{2}\right)$ & Error \\
\hline \multirow{2}{*}{$\frac{x}{\underline{x}}$} & LOS & $\begin{array}{c}(0.043,123.4) \\
U_{P E R}=95.2 \%\end{array}$ & $6.4 \%$ & $\begin{array}{l}(0.041,137) \\
U_{P E R}=91.78 \%\end{array}$ & $6.8 \%$ \\
\hline & NLOS & $\begin{array}{c}(0.068,48) \\
U_{P E R}=93.24 \%\end{array}$ & $6.7 \%$ & $\begin{array}{c}(0.051,54) \\
U_{P E R}=88.67 \%\end{array}$ & $7.9 \%$ \\
\hline \multirow{2}{*}{$\begin{array}{l}\overrightarrow{\mathscr{A}} \\
\tilde{\approx}\end{array}$} & LOS & $(-30.5,1.69)$ & $3.67 \mathrm{dBm}$ & $(-29.9,1.75)$ & $3.66 \mathrm{dBm}$ \\
\hline & NLOS & $(-33.3,2.17)$ & $3.88 \mathrm{dBm}$ & $(-34.6,2)$ & $3.8 \mathrm{dBm}$ \\
\hline
\end{tabular}

\section{CONCLUSIONS}

This paper has presented the first empirical performance model for 2-hop MCN-MR communications. The proposed model is based on a sigmoid function with the main variables being the average cellular throughput at the hybrid node, and the distance of the P2P ad-hoc link between the hybrid and destination nodes. The conducted study has shown that the model's fitting parameters heavily depend on the LOS or NLOS conditions and the communication settings of the ad-hoc link. The proposed empirical model has been derived using existing cellular (HSDPA rel. 5) and wireless (802.11g) technologies, although extending this model to other cellular technologies could be feasible as long as the MCN-MR performance is upper-bounded by the cellular link. Using the MCN-MR measurements, the paper also reports P2P link-level models derived as function of the distance between communicating nodes. The authors are currently working on extend the measurements and work to model the MCN-MR performance under configurations with an increasing number of hops between source and destination.

\section{ACKNOWLEDGMENTS}

This work was supported in part by the Spanish Ministry of Economy and Competitiveness and FEDER funds under the project TEC2011-26109, and the Local Government of Valencia with reference ACIF/2010/161. The authors also acknowledge the support of Orange Spain.

\section{REFERENCES}

[1] 3GPP TR 36.806 V9.0.0. 2010. Technical Specification Group Radio Access Network; Evolved Universal Terrestrial Radio Access (E-UTRA); Relay architectures for E-UTRA (LTE-Advanced). Mar. 2010 (Release 9).

[2] Hoymann, C., Wanshi C., Montojo, J., Golitschek, A., Koutsimanis, C., and Xiaodong S. 2012. Relaying operation in 3GPP LTE: challenges and solutions. IEEE Communications Magazine, 50, 2 (Feb. 2012), 156-162. DOI: 10.1109/MCOM.2012.6146495.

[3] Long, L., and Hossain, E. 2007. Multihop Cellular Networks: Potential Gains, Research Challenges, and a Resource Allocation Framework. IEEE Communications Magazine, 45, 9 (Sept. 2007), 66-73. DOI: 10.1109/MCOM.2007.4342859.

[4] 3GPP TR 22.803 V12.1.0. 2013. Technical Specification Group Services and System Aspects; Feasibility study for Proximity Services (ProSe). Mar. 2013 (Release 12).

[5] Wirth, T., Venkatkumar, V., Haustein, T., Schulz, E., and Halfmann, R. LTE-Advanced Relaying for Outdoor Range Extension. 2009. In Proceedings of the IEEE Vehicular Technology Conference Fall (Alaska, USA, Sept. 20-23, 2009). DOI: 10.1109/VETECF.2009.5378969.

[6] Jiansong G., Zhiheng G., Fan R., Liu W., Wang H., Sandlund, K., Liu J., Shen X., and Liu G. LTE In-Band Relay Pototype and Field Measurement. 2012. In Proceedings of the IEEE Vehicular Technology Conference Spring (Yokohama, Japan, May 6-9, 2012). DOI: 10.1109/VETECS.2012.6239938.

[7] Sharma, A., Navda, V., Ramjee, R., Padmanabhan, V.N, and Belding, E. M. 2009. Cool-Tether: energy efficient on-thefly wifi hot-spots using mobile phones. In Proceedings of the 
ACM International Conference on Emerging Networking Experiments and Technologies (Rome, Italy, Dec. 1-4, 2009). DOI=10.1145/1658939.1658952.

[8] Xinzhou Wu, Tavildar, S., Shakkottai, S., Richardson, T., Junyi Li, Laroia, R., and Jovicic, A. 2010. FlashLinQ: A synchronous distributed scheduler for peer-to-peer ad hoc networks. In Proceedings of the Annual Allerton Conference on Communication, Control, and Computing (Illinois, USA, Sept. 29 - Oct. 1, 2010). DOI: 10.1109/ALLERTON.2010.5706950.

[9] Gozalvez, J., and Coll-Perales, B. 2013. Experimental Evaluation of Multi-Hop Cellular Networks using Mobile Relays. IEEE Communications Magazine, 51, 7 (Jul. 2013), 122-129. DOI: 10.1109/MCOM.2013.6553688 .

[10] Bansal, S., Shorey, and R., Kherani, A.A. 2004. Performance of TCP and UDP protocols in multi-hop multi-rate wireless networks. In Proceedings of the IEEE Wireless Communications and Networking Conference (Georgia, USA, Mar. 21-25, 2004). DOI: 10.1109/WCNC.2004.1311548.
[11] Krishnaswamy, D. 2002. Game theoretic formulations for network-assisted resource management in wireless networks. In Proceedings of the IEEE Vehicular Technology Conference Fall (Vancouver, Canada, Mar. 21-15, 2002). DOI: 10.1109/VETECF.2002.1040428.

[12] Xia, D., Hart. J, and Fu, Q. 2013. Evaluation of the Minstrel Rate Adaptation Algorithm in IEEE 802.11g WLANs. In Proceedings of the IEEE International Conference on Communications (Budapest, Hungary, Jun. 9-13, 2013).

[13] Mare, S., Kotz, D., and Kumar, A. 2010. Experimental validation of analytical performance models for IEEE 802.11 networks. In Proceedings of the IEEE International Conference on Communication Systems and Networks (Liverpool, UK, Jan. 5-9, 2010). DOI: 10.1109/COMSNETS.2010.5431957.

[14] Capulli, F., Monti, C., Vari, M., and Mazzenga, F. 2006. Path Loss Models for IEEE 802.11a Wireless Local Area Networks. In Proceedings of the IEEE International Symposium on Wireless Communication Systems (Valencia, Spain, Sept. 6-8, 2006). DOI: 10.1109/ISWCS.2006.4362375 